\title{
Delocalization of a disordered bosonic system by repulsive interactions
}

\author{
B. Deissler ${ }^{1 \star}{ }^{,}$M. Zaccanti ${ }^{1}$, G. Roati ${ }^{1}$, C. D'Errico ${ }^{1}$, M. Fattori ${ }^{1}{ }^{2}$, M. Modugno ${ }^{1}$, G. Modugno ${ }^{1}$ \\ and $M$. Inguscio ${ }^{1}$
}

Clarifying the interplay of interactions and disorder is fundamental to the understanding of many quantum systems, including superfluid helium in porous media', granular and thin-film superconductors ${ }^{2-5}$ and light propagating in disordered media ${ }^{6-8}$. One central aspect for bosonic systems ${ }^{9-11}$ is the competition between disorder, which tends to localize particles, and weak repulsive interactions, which instead have a delocalizing effect. As the required degree of independent control of disorder and of interactions is not easily achievable in most available physical systems, a systematic experimental investigation of this competition has not so far been possible. Here we use a degenerate Bose gas with tunable repulsive interactions in a quasiperiodic lattice potential to study this interplay in detail. We characterize the entire delocalization crossover through the study of the average local shape of the wavefunction, the spatial correlations and the phase coherence. Three different regimes are identified and compared with theoretical expectations ${ }^{12-17}$ : an exponentially localized Anderson glass and the formation of locally coherent fragments as well as a coherent, extended state. These results provide insight into the role of weak repulsive interactions in disordered bosonic systems, but our approach should also enable investigations of disordered systems with interactions in the strongly correlated regime ${ }^{18-20}$.

The interplay of disorder and interactions lies at the heart of the behaviour of many physical systems. Notable examples are the transitions to insulators observed in superconductors and metals ${ }^{2-5}$, quantum Hall physics ${ }^{21}$, electrical conduction in DNA (ref. 22), or light propagation in nonlinear disordered media ${ }^{7,8}$. An important step towards their full comprehension is understanding disordered bosonic systems at zero temperature, where a competition between disorder and weak repulsive interactions is expected. Indeed, whereas disorder tends to localize non-interacting particles, giving rise to Anderson localization ${ }^{23}$, weak repulsive interactions can counteract this localization to minimize the energy. Eventually, interactions can screen the disorder and bring the system towards a coherent, extended ground state, that is, a BoseEinstein condensate (BEC). In many years of research, mainly theoretical predictions have been made about the properties of the complex phases expected to appear as a result of this competition $^{9-17}$. A systematic experimental study has not so far been possible, because on the one hand interactions in condensedmatter systems are strong but difficult to control ${ }^{1}$, and on the other hand in photonic systems only nonlinearities corresponding to attractive interactions ${ }^{7,8}$ have been explored in experiments. Instead, ultracold atoms in disordered optical potentials are a promising system for such investigations ${ }^{15,24,25}$, and have already

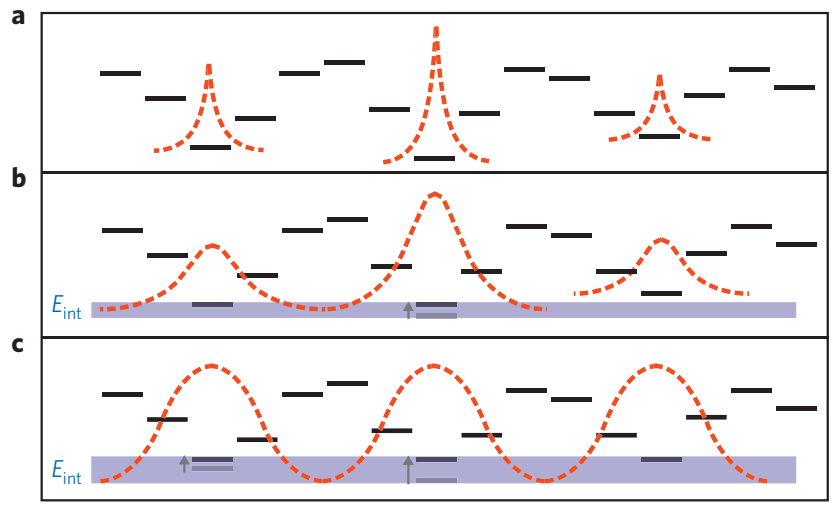

Figure 1 | Delocalization in a quasiperiodic potential. Schematic of the interaction-induced delocalization. $\mathbf{a}$, In a very weakly interacting system with sufficiently large disorder, the eigenstates are exponentially localized, and several of the lowest-energy states are populated an average of 4.4 lattice sites apart (AG). b,c, The energies of different states can become degenerate owing to repulsive interactions and their shape might be modified, giving rise to the formation of locally coherent fragments $(\mathrm{fBEC})(\mathbf{b})$, though global phase coherence is not restored until the entire system forms a coherent, extended state (BEC) at large interaction strengths (c).

enabled the observation of Anderson localization for bosons in the regime of negligible interactions ${ }^{26,27}$. Using one of these systems in a disordered lattice, we characterize the whole crossover from the regime of disorder-induced localization to that of Bose-Einstein condensation by tuning repulsive interactions in a controlled manner. The simultaneous measurement of localization properties, spatial correlations and phase coherence properties, and the comparison with the predictions of a theoretical model, enable us to identify the different regimes of this delocalization crossover.

The system used consists of a three-dimensional degenerate Bose gas of ${ }^{39} \mathrm{~K}$ in a one-dimensional quasiperiodic potential, which is generated by perturbing a strong primary optical lattice of periodicity $d=\pi / k_{1}$ with a weak secondary lattice of incommensurate periodicity $\pi / k_{2}(k=2 \pi / \lambda$, where $\lambda$ is the wavelength of the light generating the lattice). The corresponding Hamiltonian is characterized by the site-to-site tunnelling energy $J$ of the primary lattice, which is kept fixed in the experiment, and the disorder strength $\Delta$. The interatomic interactions can be controlled by changing the atomic $s$-wave scattering length $a$ by means of a Feshbach resonance ${ }^{28}$, which in turn determines the mean interaction energy per particle $E_{\text {int }}$ (see Methods). In the case of non-interacting atoms, such a

${ }^{1}$ LENS and Dipartimento di Fisica, Università di Firenze, and INO-CNR, 50019 Sesto Fiorentino, Italy, ${ }^{2}$ Museo Storico della Fisica e Centro Studi e Ricerche 'E. Fermi', 00184 Roma, Italy. *e-mail: deissler@lens.unifi.it. 
a

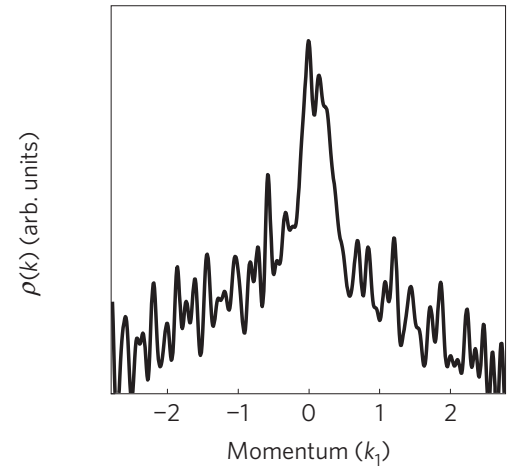

c

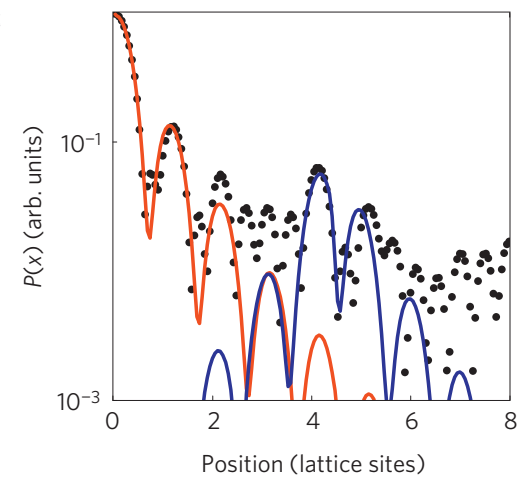

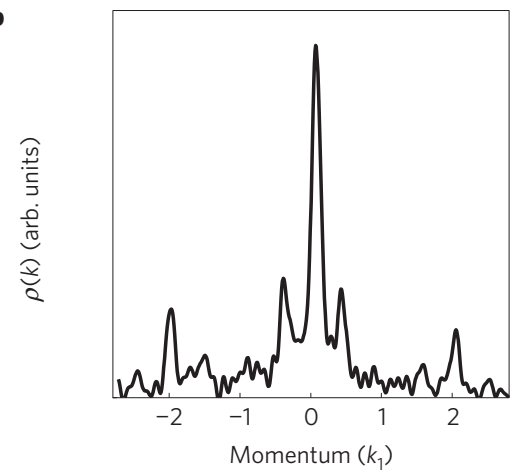

d

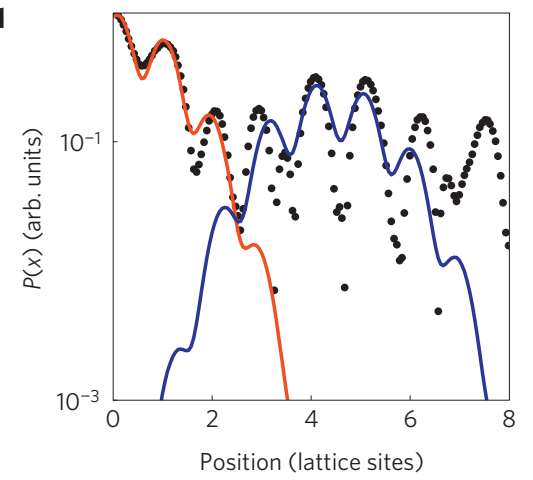

Figure 2 | Analysing the momentum distribution. a,c, Typical momentum distribution (a) and mean local shape of the wavefunction (c) recovered from a Fourier transform (FT) in the localized regime; b,d, the same in the extended regime, respectively. The root-mean-squared width of the momentum distribution and the exponent extracted from a fit (red and blue lines) to the FT give the localization properties. The coherence properties are extracted by measuring the fluctuations of the phase of the interference pattern in the momentum distribution, or by the relative height of the two states $4.4 d$ apart, which can be related to the spatially averaged correlation function $g(4.4 d)$.
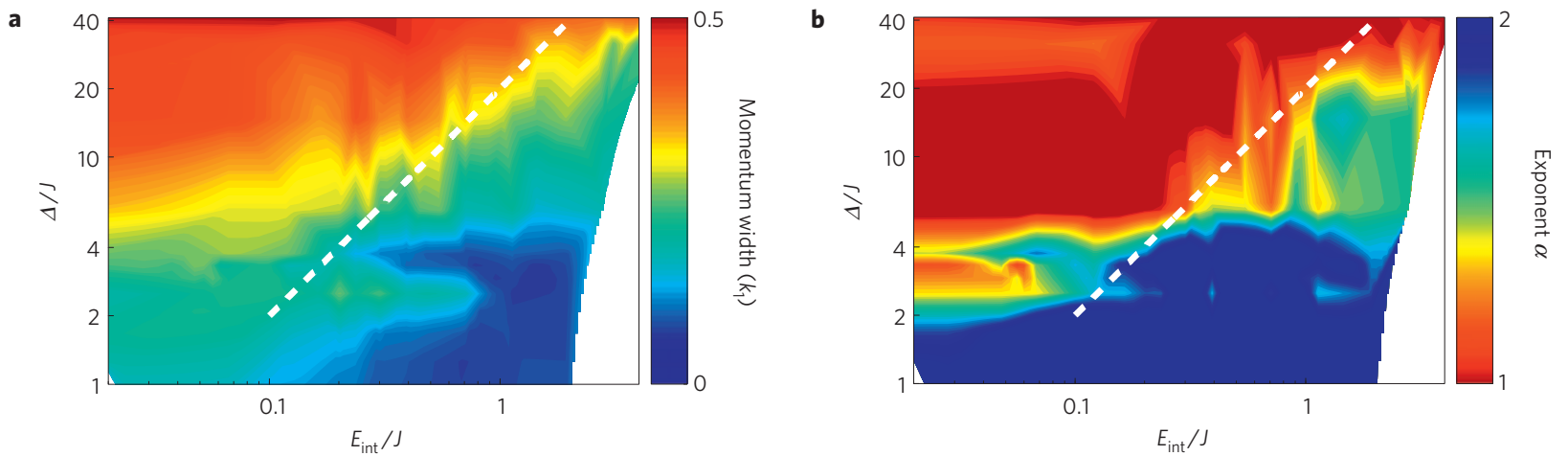

Figure 3 | Probing the interaction-induced delocalization. a, Root-mean-square width (in units of $k_{1}$ ) of the central peak of the momentum distribution. The white line gives $E_{\text {int }}=0.05 \Delta$ (standard deviation of the lowest-lying energies), where we expect the centre of the delocalization crossover. $\mathbf{b}$, Average

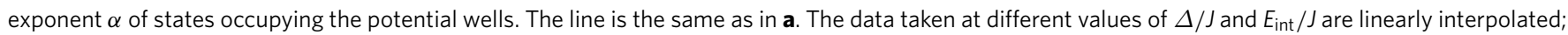
the colour indicates the mean value of the measured quantity.

system is a realization of the Aubry-André model ${ }^{29}$, which shows an Anderson-like localization transition for a finite value of the disor$\operatorname{der} \Delta / J=2$. Above the transition, the non-interacting eigenstates of the potential are exponentially localized owing to the quasiperiodic perturbation of the lattice on-site energies and the energy spectrum is split into 'minibands' ${ }^{13,30}$. The localization properties in this case have been studied experimentally in detail in ref. 27, where it was seen that several low-lying eigenstates, separated on average by $d /(\beta-1) \approx 4.4 d$, where $\beta=k_{2} / k_{1}$, are typically populated in the experiment. Adding weak interactions, the different regimes that appear as a result of the interplay of disorder and interactions can be explored. For very weak repulsive interactions, the occupation of several eigenstates in the lowest miniband is favoured (Fig. 1a). This regime, in which several exponentially localized states coexist without phase coherence, is often identified with an Anderson glass ${ }^{11,15}$ $(\mathrm{AG})$. As $E_{\text {int }}$ is increased, coherent fragments, which extend over more than one well of the quasiperiodic potential, are expected to form (Fig. 1b). In this case, global phase coherence would not yet be restored, and the local shape of the states might be modified. Some authors have called this regime a 'fragmented BEC' (fBEC; ref. 12). Finally, for large enough $E_{\text {int }}$ a single, extended phase-coherent state is expected to be formed, that is, a macroscopic BEC (Fig. 1c).

The system is prepared by first loading an interacting condensate adiabatically from the ground state of a harmonic trap into the quasiperiodic lattice. The interaction energy is then slowly changed to its final value $E_{\text {int }}$, and the confining potential is reduced. This 
process is adiabatic for most of the parameter range explored until $E_{\text {int }}$ becomes sufficiently low for the system to enter the fully localized regime. Here, several independent low-lying excited states are populated even when it would be energetically favourable to populate just the ground state. This loss of adiabaticity is seen experimentally as a transfer of energy into the radial direction (see Supplementary Information).

The system is characterized in detail by analysing its momentum distribution, which is recovered by taking an image of the condensate after a long ballistic expansion without interactions (see Methods). From the momentum distribution and derived Fourier transforms, of which we show examples in Fig. 2, we extract the local shape of the wavefunction, spatial correlations and phase coherence properties for different values of $\Delta / J$ and $E_{\text {int }} / J$. The system can be approximately described as the superposition of states with the same envelope separated by $4.4 \mathrm{~d}$. First, the mean extension of individual states can be quantified by measuring the rootmean-squared width of the momentum distribution (Fig. 2a,b). A smaller (larger) width indicates a more extended (localized) state. Next, the mean local shape of the wavefunction on a length scale of $4.4 d$ is extracted from the Fourier transform of the square root of the momentum distribution. From a fit to a generalized exponential function, the localization exponent $\alpha$ is recovered (see also Methods), as shown in Fig. 2c,d. The measured momentum width and exponent are shown in Fig. 3. We find that for very small $E_{\text {int }}$ the states are exponentially localized, because $\alpha \approx 1$, and the momentum width is large, consistent with the AG regime. Increasing $E_{\text {int }}$, the width decreases and the exponent increases to $\alpha \approx 2$. Repulsive interactions therefore delocalize the system as expected, or, alternatively, the localization crossover is shifted to higher values of the disorder strength $\Delta / J$ when interactions are introduced into the system. The position of the delocalization crossover is in good agreement with the expectations of a simple screening argument ${ }^{14}$. The increasing interaction energy serves to smooth over the disordering potential in the occupied sites, providing a flatter energetic landscape on which more extended states can form. The centre of the crossover is therefore expected to occur when $E_{\text {int }}$ is comparable to the standard deviation of energies in the lowest miniband of the non-interacting spectrum, $0.05 \Delta$ (white line in Fig. 3, see also Supplementary Information).

The correlation properties of neighbouring states can be extracted from a Fourier transform of the momentum distribution itself, which gives the spatially averaged first-order correlation function $g(x)$ (see Methods). In Fig. $4 \mathrm{a}, \mathrm{b}, g(x)$ at $x=4.4 d$ is shown for both the experiment and a ground-state theory that we have developed, with generally good agreement. In the localized regime, the correlation is exactly zero in the theory, because no neighbouring states are occupied. In contrast, the correlation is finite in the experiment owing to the occupation of neighbouring localized states arising from the non-adiabatic loading, but is small because the states are independent. As $E_{\text {int }}$ is increased, the correlation features a crossover towards larger values, signalling that coherence is progressively established locally over distances of at least $4.4 \mathrm{~d}$. The shape of the crossover in the experiment is again in qualitative agreement with the expectation of the screening argument above (see the white line in Fig. 4a).

Finally, information about the phase coherence of neighbouring states can be obtained by measuring the phase $\phi$ of the interference pattern in the momentum distribution for repeated runs of the experiment with the same parameters (see Methods for details). If the states are not phase locked, $\phi$ changes almost randomly at each repetition of the experimental sequence. In Fig. $4 \mathrm{~d}$ we show the standard deviation of $\phi$, estimated from a large number of repetitions of the experiment, for fixed $\Delta / J=12$. We see a slight decrease of the phase fluctuations with increasing $E_{\text {int }}$, which nevertheless remain relatively large in the crossover region where
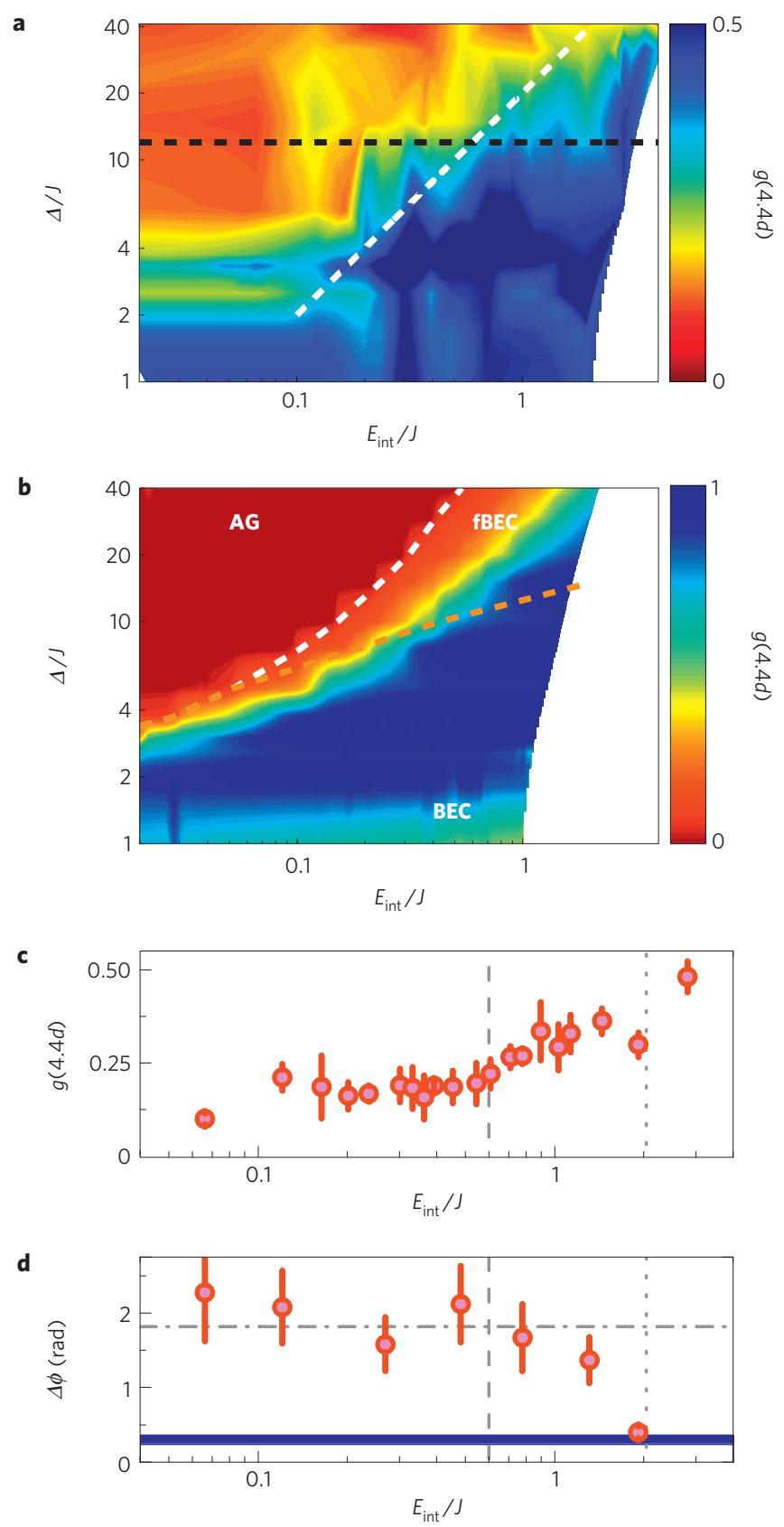

Figure 4 | Probing the phase coherence of the system. $\mathbf{a}, \mathbf{b}$, Correlation of neighbouring localized states. a, Experiment (white line as in Fig. 3).

b. Theory of the ground state. The white and orange dashed lines show the boundaries between an AG, an $\mathrm{ABEC}$ and a macroscopic BEC. c, Correlation of neighbouring states for $\Delta / J=12$, corresponding to the black dashed line in $\mathbf{a}$. The error bar is given by the standard error of the mean. $\mathbf{d}$, Standard deviation of the phase measured by repeating the experiment up to 25 times for a given set of parameters for $\Delta / J=12$. The error is estimated as $\Delta \phi / \sqrt{N}$, where $N$ is the number of images from which the phase was extracted. The blue bar shows the phase fluctuations measured for an extended system below the localization threshold. The grey dash-dotted line gives the standard deviation for a purely random distribution. In c and $\mathbf{d}$, the grey dashed (dotted) lines give $E_{\text {int }}=0.05 \Delta(0.17 \Delta)$.

the correlation increases (Fig. 4c). The fluctuations finally drop to the background value only when $E_{\text {int }}$ is comparable to the full width of the lowest miniband of the non-interacting spectrum, $0.17 \Delta$. These observations confirm that in the localized regime the states are totally independent, which together with the localization 
properties (Fig. 3) indicates that the system can indeed be described as an AG (refs 11,15). The system crosses a large region of only partial coherence while becoming progressively less localized as $E_{\text {int }}$ is increased. This is consistent with the formation of locally coherent fragments expected for an fBEC. An analogous fragmentation behaviour was reported in ref. 31 . Finally, the features of a single extended, coherent state are seen, that is, a BEC.

In the mean-field theory, boundaries between the different regimes expected for the system can be defined (see Methods). In particular, the crossover from the AG phase to an $\mathrm{ABEC}$ (white line in Fig. $4 \mathrm{~b}$ ) occurs when $g(4.4 d)$ starts to increase. Similarly, the orange line in Fig. $4 \mathrm{~b}$ shows where the fragments are locked together in phase to form a single macroscopic condensate for very large interactions. The generally good agreement between the experimental observables and theory indicates that our system is well described by the mean-field theory for most of the parameter space explored experimentally.

In conclusion, we have provided the first experimental characterization of the localization, correlation and coherence properties of the various regimes owing to the competition of disorder and weak repulsive interactions in a bosonic system. Other aspects of the delocalization crossover worth further study are, for example, the detailed properties of the ground state of the AG regime, which was not possible to study in the present set-up, and the presence of a superfluid-insulator transition at the BEC-fBEC boundary analogous to the one observed in superconductors ${ }^{2}$. Regarding the latter, in transport experiments analogous to the ones described in ref. 27 we have been able to verify that the AG and $\mathrm{ABEC}$ regimes are not inconsistent with being insulating, as is the case in the regime of vanishing $E_{\text {int }}$ (see Supplementary Information). Finally, it would be appealing to use the present system and the correlation analysis introduced here to explore the regime of strong correlations, $E_{\text {int }} \gg J$, which could be reached by using a quasi-one-dimensional system with strong radial confinement. There, another elusive insulating phase due to the cooperation of disorder and interactions, the so-called Bose-glass phase, is expected to appear, although there is debate on the exact shape of the phase diagram ${ }^{11,15,18,19,32}$.

\section{Methods}

Condensate with tunable interactions. $\mathrm{A}^{39} \mathrm{~K}$ condensate of about $N=20,000$ atoms with an $s$-wave scattering length of $250 a_{0}$, where $a_{0}=52.9 \mathrm{pm}$ is the Bohr radius, is prepared in a harmonic optical trap. The condensate is loaded into the quasiperiodic potential while the optical trap is decompressed in about $250 \mathrm{~ms}$ to reduce the harmonic confinement, and a gravity-compensating magnetic-field gradient is added. At the same time, the scattering length $a$ is changed by means of a broad Feshbach resonance to values ranging from $a \leq 0.1 a_{0}$ to about $a=300 a_{0}$ (ref. 28 )

Quasiperiodic potential. The quasiperiodic potential is created by two vertically oriented laser beams in standing-wave configuration. The primary lattice is generated by a Nd:YAG laser with a wavelength of $\lambda_{1}=1,064.4 \mathrm{~nm}$ and has a strength of $s_{1}=V_{1} / E_{\mathrm{R}, 1}=10.5$ (corresponding to $J / h=79 \mathrm{~Hz}$ ), as measured in units of the recoil energy $E_{\mathrm{R}, 1}=h^{2} /\left(2 M \lambda_{1}^{2}\right)$. The secondary lattice is generated by a Ti:sapphire laser of wavelength $\lambda_{2}=866.6 \mathrm{~nm}$, the strength being adjustable up to $s_{2}=V_{2} / E_{\mathrm{R}, 2}=1.7$. Both beams are focused onto the condensate with a beam waist of about $150 \mu \mathrm{m}$. The lattice lasers give a harmonic confinement of $\omega_{\perp}=2 \pi \times 50 \mathrm{~Hz}$ in the radial direction. In the vertical (axial) direction, a weak confinement of $5 \mathrm{~Hz}$ is given by a weak optical trap as well as by a curvature from the gravity-compensating magnetic field.

Energy scales. In the tight-binding limit, the hopping energy $J$ and disorder strength $\Delta$ can be estimated as $J=1.43 s_{1}^{0.98} \exp \left\{-2.07 \sqrt{s_{1}}\right\} E_{\mathrm{R}, 1}$ and $\Delta=0.5 s_{2} \beta^{2}\left(1.0264 \exp \left\{-2.3624 / s_{1}^{0.59}\right\}\right) E_{\mathrm{R}, 1}$ (ref. 30). The experimental uncertainty on $\Delta / J$ is around $15 \%$. We estimate that around 30 lattice sites, corresponding to about seven localized states, are populated during the loading of the lattice. We then define a mean interaction energy per particle $E_{\text {int }}=g N / 7 \int|\varphi(\mathbf{r})|^{4} \mathrm{~d}^{3} \mathbf{r}$, where $g=4 \pi \hbar^{2} a / m, m$ is the atomic mass and $\varphi(\mathbf{r})$ is a Gaussian approximation to the on-site Wannier function. We include coupling in the radial directions of our system, with the consequence that the interaction energy is nonlinear in the scattering length. Though this definition of the energy is strictly valid only in the localized regime, comparison with a numerical simulation of our experimental procedure has shown that it is a good approximation for all values of the scattering length up to an error of $30 \%$. Note that the potential energy from the residual harmonic confinement is approximately $3 \times 10^{-3} \mathrm{~J}$ over a distance $4.4 d$.

Momentum-distribution analysis. The images of the momentum distribution are taken by absorption imaging with a CCD (charge-coupled device) camera after $36.5 \mathrm{~ms}$ ballistic expansion. At the time of release, the scattering length is set to below $1 a_{0}$ in less than $1 \mathrm{~ms}$ and kept there until the Feshbach magnetic field is switched off $10 \mathrm{~ms}$ before taking the image - at this point, the system has expanded a sufficient amount to minimize the effect of interactions. For such a free expansion, the image is approximately the in-trap momentum distribution $\rho(k)=\left\langle\hat{\Psi}^{\dagger}(k) \hat{\Psi}(k)\right\rangle$. The acquired images are integrated along the radial direction to obtain a profile. In momentum space, the width of the central peak is calculated by taking the root-mean-square width within the first Brillouin zone. Owing to the quasiperiodic lattice potential, for a sufficiently homogeneous system the in-trap wavefunction can be decomposed into copies of a single state with real and non-negative envelope $\xi(x) \sim \exp \left(-|x / L|^{\alpha}\right)$, spaced by $4.4 d$. Therefore, in momentum space, $\sqrt{\rho(k)}=\xi(k) \mathcal{S}(k)$, where $\mathcal{S}(k)$ is an interference term, and $\xi(x)$ can be extracted from a Fourier transform of $\sqrt{\rho(k)}$ (see also Supplementary Information). We fit to the sum of two generalized exponential functions $A \exp \left(-\left|\left(x-x_{\mathrm{c}}\right) / L\right|^{\alpha}\right) \cdot\left[1+B \cos \left(k_{1}\left(x-x_{\mathrm{c}}\right)+\delta\right)\right]$, where $x_{\mathrm{c}}$ denotes the centre of each of these functions, spaced by $4.4 d$. From this fit, the exponent $\alpha$ is recovered. In addition, from the Wiener-Khinchin theorem, the momentum distribution can be expressed in terms of the first-order correlation function $G\left(x^{\prime}, x+x^{\prime}\right)=\left\langle\hat{\Psi}^{\dagger}\left(x^{\prime}\right) \hat{\Psi}\left(x+x^{\prime}\right)\right\rangle$ as $\rho(k) \propto \mathfrak{F}^{-1} \int G\left(x^{\prime}, x+x^{\prime}\right) \mathrm{d} x^{\prime}$. By taking the Fourier transform of the momentum distribution itself, we can therefore recover the spatially averaged correlation function $g(x)=\int G\left(x^{\prime}, x+x^{\prime}\right) \mathrm{d} x^{\prime}$. With the same fitting function as above, we evaluate the spatially averaged correlation between two states 4.4 lattice sites apart, $A_{2} / A_{1}$. Experimentally, the correlation function saturates at a value around 0.5 owing to the finite momentum resolution. The fluctuations in phase between neighbouring states are seen as a fluctuation of the phase $\phi$ of the interference pattern of the momentum distribution, which is directly extracted from a fit (see also Supplementary Information). The two-dimensional graphs in Figs 3 and 4 were generated by linearly interpolating a total of 130 averaged datapoints at nine different values of disorder, changing the interactions. Typical experimental scatter and statistical errors are seen in Fig. 4c.

Theory of the ground state. The theoretical calculations presented in the paper rely on a mean-field approach. This is an effective one-dimensional model, which also partially includes the radial-to-axial coupling, and is known to provide an accurate description in the two limiting cases of Anderson localization and a BEC. The boundaries between the different regimes shown in Fig. $4 \mathrm{~b}$ are obtained by analysing the correlation function $g(x)$ and the density distribution. In the theory we define the AG phase as the one in which the correlation $g(4.4 d)$ is zero. To enter the fBEC phase, we require $g(4.4 d)>0$, which implies that coherent fragments composed of adjacent localized states can start to form. For increasing $E_{\text {int }}$ the extension of the fragments increases, until most of the system remains in a single component, which corresponds to a macroscopic BEC. To define the boundary between the $\mathrm{ABEC}$ and the $\mathrm{BEC}$ regimes, we first identify as fragments the parts of the system separated by low-density regions for which an applied relative phase twist does not affect the energy of the system. When one single macroscopic fragment forms, we assume the system to be in the BEC regime. A more detailed description of the theoretical methods can be found in the Supplementary Information.

Received 19 October 2009; accepted 3 March 2010; published online 11 April 2010

\section{References}

1. Reppy, J. D. Superfluid helium in porous media. J. Low Temp. Phys. 87, 205-245 (1992).

2. Dubi, Y., Meir, Y. \& Avishai, Y. Nature of the superconductor-insulator transition in disordered superconductors. Nature 449, 876-880 (2007).

3. Beloborodov, I. S., Lopatin, A. V., Vinokur, V. M. \& Efetov, K. B. Granular electronic systems. Rev. Mod. Phys. 79, 469-518 (2007).

4. Goldman, A. M. \& Marković, N. Superconductor-insulator transitions in the two-dimensional limit. Phys. Today 51, 39-44 (1998).

5. Phillips, P. \& Dalidovich, D. The elusive Bose metal. Science 302, 243-247 (2003).

6. Pertsch, T. et al. Nonlinearity and disorder in fibre arrays. Phys. Rev. Lett. 93, 053901 (2004).

7. Schwartz, T., Bartal, G., Fishman, S. \& Segev, M. Transport and Anderson localization in disordered two-dimensional photonic lattices. Nature 446, 52-55 (2007).

8. Lahini, Y. et al. Anderson localization and nonlinearity in one-dimensional disordered photonic lattices. Phys. Rev. Lett. 100, 013906 (2008).

9. Giamarchi, T. \& Schulz, H. J. Anderson localization and interactions in one-dimensional metals. Phys. Rev. B 37, 325-340 (1988). 
10. Fisher, D. S. \& Fisher, M. P. A. Onset of superfluidity in random media. Phys. Rev. Lett. 61, 1847-1850 (1988).

11. Scalettar, R. T., Batrouni, G. G. \& Zimanyi, G. T. Localization in interacting, disordered, Bose systems. Phys. Rev. Lett. 66, 3144-3147 (1991).

12. Lugan, P. et al. Ultracold Bose gases in 1D disorder: From Lifshits glass to Bose-Einstein condensate. Phys. Rev. Lett. 98, 170403 (2007).

13. Roux, G. et al. Quasiperiodic Bose-Hubbard model and localization in one-dimensional cold atomic gases. Phys. Rev. A 78, 023628 (2008).

14. Lee, D. K. K. \& Gunn, J. M. F. Bosons in a random potential: Condensation and screening in a dense limit. J. Phys. Condens. Matter 2, 7753-7768 (1990).

15. Damski, B., Zakrzewski, J., Santos, L., Zoller, P. \& Lewenstein, M. Atomic Bose and Anderson glasses in optical lattices. Phys. Rev. Lett. 91, 080403 (2003).

16. Schulte, T. et al. Routes towards Anderson-like localization of Bose-Einstein condensates in disordered optical lattices. Phys. Rev. Lett. 95, 170411 (2005).

17. Falco, G. M., Nattermann, T. \& Pokrovsky, V. L. Localized states and interaction-induced delocalization in Bose gases with quenched disorder. Europhys. Lett. 85, 30002 (2009).

18. Fisher, M. P. A., Weichman, P. B., Grinstein, G. \& Fisher, D. S. Boson localization and the superfluid-insulator transition. Phys. Rev. B 40, 546-570 (1989).

19. Fallani, L., Lye, J. E., Guarrera, V., Fort, C. \& Inguscio, M. Ultracold atoms in a disordered crystal of light: Towards a Bose glass. Phys. Rev. Lett. 98, 130404 (2007).

20. Pasienski, M., McKay, D., White, M. \& DeMarco, B. Disordered insulator in an optical lattice. Preprint at http://arxiv.org/abs/0908.1182 (2009).

21. Martin, J. et al. The nature of localization in graphene under quantum Hall conditions. Nature Phys. 5, 669-674 (2009).

22. Endres, R. G., Cox, D. L. \& Singh, R. R. P. Colloquium: The quest for high-conductance DNA. Rev. Mod. Phys. 76, 195-214 (2004).

23. Anderson, P. W. Absence of diffusion in certain random lattices. Phys. Rev. 109, 1492-1505 (1958)

24. Fallani, L., Fort, C. \& Inguscio, M. Bose-Einstein condensates in disordered potentials. Adv. At. Mol. Opt. Phys. 56, 119-160 (2008).

25. Sanchez-Palencia, L. \& Lewenstein, M. Disordered quantum gases under control. Nature Phys. 6, 87-95 (2010).
26. Billy, J. et al. Direct observation of Anderson localization of matter waves in a controlled disorder. Nature 453, 891-894 (2008).

27. Roati, G. et al. Anderson localization of a non-interacting Bose-Einstein condensate. Nature 453, 895-898 (2008).

28. Roati, G. et al. ${ }^{39} \mathrm{~K}$ Bose-Einstein condensate with tunable interactions. Phys. Rev. Lett. 99, 010403 (2007).

29. Aubry, S. \& André, G. Analycity breaking and Anderson localization in incommensurate lattices. Ann. Israel Phys. Soc. 3, 133-140 (1980).

30. Modugno, M. Exponential localization in one-dimensional quasi-periodic optical lattices. New J. Phys. 11, 033023 (2009).

31. Chen, Y. P. et al. Phase coherence and superfluid-insulator transition in a disordered Bose-Einstein condensate. Phys. Rev. A 77, 033632 (2008).

32. Pollet, L., Prokof ev, N. V., Svistunov, B. V. \& Troyer, M. Absence of a direct superfluid to Mott insulator transition in disordered Bose systems. Phys. Rev. Lett. 103, 140402 (2009).

\section{Acknowledgements}

We thank B. Altshuler and M. Larcher for discussions, S. Müller for experimenta contributions and all the colleagues of the Quantum Gases group at LENS. This work has been supported by the EU (MEIF-CT-2004-009939), by the ESA (SAI 20578/07/NL/UJ), by the ERC through the Starting Grant QUPOL and by the ESF and INO-CNR through the EuroQUASAR program.

\section{Author contributions}

B.D., G.R. and M.Z. carried out the experiments; B.D. and C.D. analysed data; M.F. contributed to the experiment; M.M. carried out the theoretical calculations; B.D wrote the manuscript; M.I. and G.M. codirected the project; all authors participated in discussions on the manuscript.

\section{Additional information}

The authors declare no competing financial interests. Supplementary information accompanies this paper on www.nature.com/naturephysics. Reprints and permissions information is available online at http://npg.nature.com/reprintsandpermissions. Correspondence and requests for materials should be addressed to B.D. 